\title{
Active Mode Locking With Hybrid Lasers
}

\author{
Bo Guo, Li Yan, Member, IEEE, and Curtis R. Menyuk, Fellow, IEEE
}

\begin{abstract}
We present the results of a numerical study of active mode locking with hybrid lasers which contain an inhomogeneously broadened laser medium and a homogeneously broadened laser medium. The spectral, pulse, and gain characteristics of actively mode-locked hybrid lasers and the influence of the unsaturated gain, the saturation power, and the homogeneous linewidth on the pulse coherence and bandwidth are studied. The simulations show that coherent and shorter pulses are generated as compared to that by either an inhomogeneously broadened medium or a homogeneously broadened medium alone. Varying the unsaturated gain or the saturation power of the gain medium are two equivalent ways to obtain the same maximal coherent pulse bandwidth for given gain media. When different gain media can be selected, a larger pulse bandwidth can be obtained with the use of a broadband homogeneously broadened medium.
\end{abstract}

Index Terms-Lasers, mode-locked lasers, numerical analysis, optical pulse generation, ultrafast optics.

\section{INTRODUCTION}

B ETTER laser performance can be obtained by constructing a hybrid laser which contains two laser media in a single cavity to combine the best properties of both laser media together. This improvement has been demonstrated in a two-section gain medium $\mathrm{CO}_{2}$ lasers and a hybrid $\mathrm{Nd}$ : YLF and $\mathrm{Nd}$ : phosphate glass laser [1]-[3]. In the generation of ultrashort laser pulses by mode locking, there are two essential requirements. One is the creation of a broad lasing bandwidth. The other is the maintenance of good phase coherence among the lasing axial modes. Not every laser medium can provide both properties. For example, a narrow-band homogeneously broadened laser, such as the Nd:YLF or Nd:YAG, ensures good phase coherence in active mode locking, but the narrow gain linewidth limits the pulse duration. On the other hand, a broad-band inhomogeneously broadened laser, such as $\mathrm{Nd}$ : glass laser, does not suffer much from gain narrowing. It has a large lasing bandwidth even in the continuous-wave (CW) state and allows for generation of femtosecond pulses [4]. However, in active mode locking, good phase coherence is generally difficult to attain among the whole lasing bandwidth, leading to broad and partially coherent pulses [5], [6]. A large $\mathrm{CW}$ lasing bandwidth also makes it difficult for passive mode

Manuscript received February 27, 2001; revised June 12, 2001. This work was supported by the National Science Foundation under Grant ECS-9703988.

B. Guo was with the Department of Computer Science and Electrical Engineering, University of Maryland, Baltimore County, Baltimore, MD 21250. He is now with Celion Networks, Inc., Richardson, TX 75082 USA.

L. Yan is with the Department of Computer Science and Electrical Engineering, University of Maryland, Baltimore County, Baltimore, MD 21250 USA.

C. R. Menyuk is with the Department of Computer Science and Electrical Engineering, University of Maryland, Baltimore County, Baltimore, MD 21250 USA and also with the Laboratory for Telecommunications Sciences, C/O USARL, Adelphi Lab Center, Adelphi, MD 20783-1197 USA.

Publisher Item Identifier S 0018-9197(01)08333-6. locking to start up [7]-[9]. To utilize the large gain linewidth of a broad-band, inhomogeneously broadened medium without suffering from the difficulties related to its large CW lasing bandwidth, a novel approach of mode locking with a hybrid laser was proposed and first demonstrated in active mode locking with a hybrid Nd:YLF and Nd: phosphate glass laser [3]. It was shown that good phase coherence can be attained by the spectral control of $\mathrm{Nd}$ : YLF so that coherent and shorter pulses are obtained than that with $\mathrm{Nd}$ : phosphate glass alone, while a broader pulse bandwidth can be obtained in the hybrid $\mathrm{Nd}$ laser than from the Nd: YLF laser alone. The experimental results show the advantage of active mode locking with hybrid lasers over that with an inhomogeneously broadened medium, or a homogeneously broadened medium alone. In this paper, we present the results of a comprehensive numerical study of active mode locking with hybrid lasers. In Section II, we present the laser model, and we extend the coupled-mode equations to a hybrid laser which contains a homogeneously broadened gain medium and an inhomogeneously broadened gain medium. In Section III, we show the spectral, pulse, and gain characteristics of actively mode-locked hybrid lasers and discuss the parameter dependence of the pulse bandwidth and coherence of actively mode-locked hybrid lasers. Conclusions of the numerical simulation results are given in Section IV.

\section{THE MODEL}

The model of the hybrid laser that we use here is closely related to the model that was previously used in a study of CW lasing of hybrid lasers [10]. A hybrid laser contains a partially inhomogeneously broadened medium $a$ and a homogeneously broadened medium $b$. Amplitude modulation (AM) is used to actively modelock the hybrid laser. We apply the coupled-mode equations [11] to the hybrid laser. The complex electric field $\tilde{E}_{n}$ of the $n$th lasing mode and the gains of the two laser media are governed by the following coupled ordinary differential equations:

$$
\begin{aligned}
\frac{d \tilde{E}_{n}}{d t}= & -\frac{\delta_{c, n}}{2 T_{R}} \tilde{E}_{n}+\frac{1}{2 T_{R}}\left[\gamma_{a}\left(\nu_{n}\right) p_{m, a}+\gamma_{b}\left(\nu_{n}\right) p_{m, b}\right] \tilde{E}_{n} \\
& -j\left(\omega_{n}-\omega_{q}\right) \tilde{E}_{n} \\
& +\frac{\Delta_{m}}{2 T_{R}}\left(\tilde{E}_{n+1}-2 \tilde{E}_{n}+\tilde{E}_{n-1}\right)+\frac{\Delta \tilde{E}_{\mathrm{sp}}}{T_{R}} \\
\frac{d N_{a}\left(\nu_{\xi}\right)}{d t}= & \frac{N_{0 a}\left(\nu_{\xi}\right)}{\tau_{a}}-\frac{N_{a}\left(\nu_{\xi}\right)}{\tau_{a}} \\
& -\frac{N_{a}\left(\nu_{\xi}\right)}{\tau_{a}} \sum_{\nu_{n}} \frac{2 I_{a}\left(\nu_{n}\right)}{I_{s, a}} \bar{g}\left(\nu_{\xi}, \nu_{n}\right) \\
\frac{d N_{b}}{d t}= & \frac{N_{0 b}}{\tau_{b}}-\frac{N_{b}}{\tau_{b}}-\frac{N_{b}}{\tau_{b}} \sum_{\nu_{n}} \frac{2 I_{b}\left(\nu_{n}\right)}{I_{s, b}} \bar{g}\left(\nu_{b}, \nu_{n}\right)
\end{aligned}
$$


where $\gamma_{a}, p_{m, a}$, and $\gamma_{b}, p_{m, b}$ are the gain coefficient and round-trip medium length, and $N_{0 a}\left(\nu_{\xi}\right), N_{a}\left(\nu_{\xi}\right)$ and $N_{0 b}, N_{b}$ are the unsaturated and saturated population inversion densities of medium $a$ and medium $b$, respectively. The parameters $\delta_{c, n}$ and $T_{\mathrm{R}}$ are the round-trip cavity loss and cavity round-trip time, respectively. The round-trip amplitude transmission function in the AM modulator is

$\tilde{t}_{\mathrm{am}}=\exp \left[-\Delta_{m}\left(1-\cos \omega_{m} t\right)\right] \approx 1-\Delta_{m}\left(1-\cos \omega_{m} t\right)$

where $\Delta_{m}$ is the modulation index.

In (1), the frequency $\omega_{q}$ is the cavity resonant or "natural" frequency and $\omega_{n}$ is the sideband or "forced" frequency under modulation. They are defined as

$$
\begin{aligned}
& \omega_{q}=\omega_{0}+q \Delta \omega_{\mathrm{ax}} \\
& \omega_{n}=\omega_{0}+n \omega_{m}
\end{aligned}
$$

where $\omega_{m}$ is the modulation frequency. The quantity $\left(\omega_{n}-\right.$ $\left.\omega_{q}\right) T_{R}$ is the phase drift of the $n$th mode caused by dispersion in one round trip and can be expressed as

$$
\begin{aligned}
\left(\omega_{n}-\omega_{q}\right) T_{R}= & \frac{1}{2} k^{\prime \prime} p_{m}\left(\omega_{n}-\omega_{0}\right)^{2}-\frac{1}{2} \gamma_{b}\left(\nu_{n}\right) p_{m, b} \frac{2\left(\nu_{n}-\nu_{b}\right)}{\Delta \nu_{h, b}} \\
& -\sum_{\nu_{\xi}} \frac{1}{2} \gamma_{a}\left(\nu_{n}, \nu_{\xi}\right) p_{m, a} \frac{2\left(\nu_{n}-\nu_{\xi}\right)}{\Delta \nu_{h, a}}
\end{aligned}
$$

where the first term accounts for the nonresonant quadratic dispersion, or the group velocity dispersion (GVD), and the second and third terms are due to the resonant dispersion caused by media $b$ and $a$, respectively. The nonresonant dispersion does not vary much for different lasers, while the resonant dispersion can be distinctly different in different kind of lasers. The influence of dispersion on active mode locking depends on the type and magnitude of dispersion, as well as the lasing bandwidth. After removing the linear portion that gives rise to a net group delay, the round-trip phase drift due to the resonant dispersion is nearly zero around the center frequency and increases drastically beyond that flat region. For a homogeneously broadened laser, the pulse spectrum is always within the flat region, and the resonant dispersion need not be considered. Inhomogeneously broadened lasers usually have large lasing bandwidth. When the phase drift due to the resonant dispersion becomes comparable to that due to GVD within the lasing bandwidth, the resonant dispersion must be considered. In an actively mode-locked hybrid laser, when the spectrum is controlled by the homogeneously broadened medium and the lasing bandwidth is narrow, the influence of the resonant dispersion of the inhomogeneously broadened medium can be further neglected. This situation holds for most of the parameter ranges in this study. Over the lasing bandwidth, the round-trip phase drift due to the resonant dispersion is less than $3 \times 10^{-6}$ radians, while the round-trip phase drift due to the GVD reaches $\sim 3.4 \times 10^{-5}$ for $k^{\prime \prime} p_{m}=2000 \mathrm{fs}^{2}$, which is a typical value for solid-state lasers and is used in our simulation. Only when the GVD is compensated significantly does the influence of resonant dispersion become comparable to the GVD and have to be taken into account.
The term $\Delta \tilde{E}_{\mathrm{sp}}$ is the mean change of the electric field due to spontaneous emission in one round trip, which is given by

$$
\Delta \tilde{E}_{\mathrm{sp}}=\sqrt{\gamma_{a}\left(\nu_{n}\right) p_{m, a}+\gamma_{b}\left(\nu_{n}\right) p_{m, b}} \sqrt{I_{\mathrm{sp}}} \exp \left[i \phi_{\mathrm{sp}}\left(\nu_{n}\right)\right]
$$

where $I_{\mathrm{sp}}$ is the equivalent intensity due to a single photon and $\phi_{\mathrm{sp}}\left(\nu_{n}\right)$ is a set of random phases.

The steady-state solutions can be obtained by numerically simulating the time evolution of $\tilde{E}\left(\nu_{n}\right), N_{a}\left(\nu_{\xi}\right)$, and $N_{b}$ in successive round trips to obtain a converged solution. The spectrum and power of the laser pulse are given by calculating $\left|\tilde{E}_{n}\right|^{2}$ and $\sum_{n}\left|\tilde{E}_{n}\right|^{2}$, respectively. The Fourier transform of $\tilde{E}\left(\nu_{n}\right)$ gives the temporal shape of the laser pulse. The initial conditions are the unsaturated population inversion densities $N_{0, a}\left(\nu_{\xi}\right), N_{0, b}$, and the spontaneous emission field $\Delta \tilde{E}_{\mathrm{sp}}$ for $N_{a}\left(\nu_{\xi}\right), N_{b}$, and $\tilde{E}_{n}$, respectively.

\section{Simulation RESUltS AND DisCUSSION}

To simplify the numerical simulation without neglecting the main features of the hybrid lasers, we assume that both media $a$ and $b$ have the same center frequency, $\nu_{a}=\nu_{b}=\nu_{0}$. The homogeneous linewidth of medium $a$ is chosen to be $\Delta \nu_{h, a}=360$ GHz. The frequency spacing of atom packets is $\Delta \nu_{\xi} / \Delta \nu_{h, a}=$ 0.1 , and the inhomogeneity is $\Delta \nu_{i h, a} / \Delta \nu_{h, a}=2.5$ or 5.0. The linewidth of homogeneous medium $b$ ranges in $\Delta \nu_{h, b}=0.25-$ $4.0 \Delta \nu_{h, a}$ in order to study the influence of $\Delta \nu_{h, b}$ on the pulse bandwidth and coherence. The axial mode spacing is $\Delta \nu_{a x}=2$ $\mathrm{GHz}$. The laser intensity is normalized by $I_{s, a}$, the saturation intensity of medium $a$. The normalized spontaneous emission intensity is chosen as $I_{\mathrm{sp}} / I_{s, a} \approx 10^{-10}$, a typical value for solid-state lasers. The cavity loss is $\delta_{c}=0.1$. The round-trip GVD is $k^{\prime \prime} p_{m}=2000 \mathrm{fs}^{2}$. At $f_{m}=\Delta \nu_{\mathrm{ax}}=2 \mathrm{GHz}$, the modulation index is $\Delta_{m}=0.00125$. The temporal modulation curvature of this modulator is the same as that of a modulator with $f_{m}=100 \mathrm{MHz}$, and $\Delta_{m}=0.5$, typical values for actively mode-locked solid-state lasers. Numerical simulations also show that with the same dispersion, the spectra and axial mode phases are almost identical for a short cavity laser with $f_{m}=2 \mathrm{GHz}$ and $\Delta_{m}=0.00125$ and for a long cavity laser with $f_{m}=100 \mathrm{MHz}$ and $\Delta_{m}=0.5$. Under the fast gain approximation [10], the laser power becomes stable after $10^{2}$ to $10^{3}$ round trips, and the spectra and phases become stable after $10^{\tilde{5}}$ round trips for the hybrid lasers.

\section{A. Spectral and Pulse Characteristics}

In active mode locking, the modulator attempts to broaden the spectrum and maintain phase coherence among lasing modes in order to counteract the gain narrowing and phase drift caused by dispersion. In the case of an inhomogeneously broadened laser, the saturated gain is flat, and there is no gain narrowing. The modulator only competes with the dispersion. If we consider only GVD, there exists a GVD-limited, AM-lockable pulse width and bandwidth, which are given by [6]

$$
t_{p 0}=\left(\frac{2^{3 / 2} \ln 2}{\pi}\right)^{1 / 2}\left(\frac{k^{\prime \prime} p_{m}}{\Delta_{m} f_{m}^{2}}\right)^{1 / 4}
$$



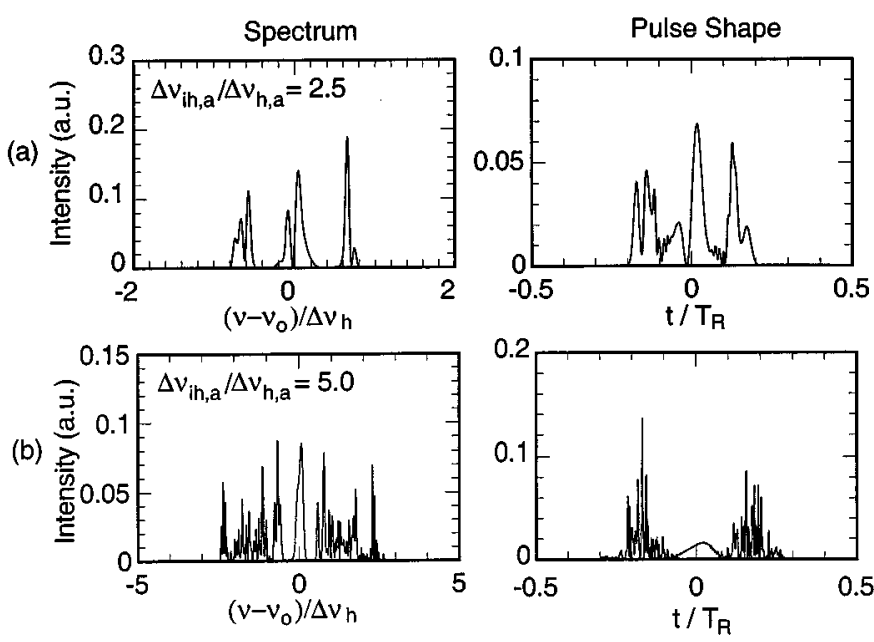

Fig. 1. Spectra and pulse shapes of actively mode-locked inhomogeneously broadened lasers with inhomogeneity of: (a) 2.5 and (b) 5.0 , respectively. The modulation frequency and modulation index are $f_{m}=2 \mathrm{GHz}$ and $\Delta_{m}=$ 0.00125 . The round-trip GVD is $k^{\prime \prime} p_{m}=2000 \mathrm{fs}^{2}$.

$$
\Delta \nu_{p 0}=\left(\frac{2^{3 / 2} \ln 2}{\pi}\right)^{1 / 2}\left(\frac{\Delta_{m} f_{m}^{2}}{k^{\prime \prime} p_{m}}\right)^{1 / 4}
$$

When the lasing bandwidth sustained by the gain medium is smaller than $\Delta \nu_{p 0}$, the pulse is fully coherent. A homogeneously broadened laser always falls in this regime due to the strong gain narrowing [6]. When the lasing bandwidth $\Delta \nu_{p}$ is larger than $\Delta \nu_{p 0}$, the modulator cannot lock all the lasing modes, resulting in partially coherent pulses. The pulse energy in the form of partially correlated or uncorrelated spikes, spreads over a range characterized by an envelope duration. With the magnitude of the modulation and dispersion used in our simulation $\left(f_{m}=2 \mathrm{GHz}, \Delta_{m}=0.00125\right.$, and $k^{\prime \prime} p_{m}=$ $2000 \mathrm{fs}^{2}$ ), the GVD-limited pulse width and bandwidth are $t_{p 0}=19.8 \mathrm{ps}$, and $\Delta \nu_{p 0}=31.4 \mathrm{GHz}$. In numerical simulation, we normalize the pulse width to the cavity round-trip time $T_{\mathrm{R}}$ and the pulse bandwidth by the homogeneous linewidth $\Delta \nu_{h, a}$. For convenience of comparison, we express $t_{p 0}=0.0397 T_{\mathrm{R}}$ and $\Delta \nu_{p 0}=0.0873 \Delta \nu_{h, a}$.

Fig. 1 shows the spectra and pulse shapes of the actively mode-locked inhomogeneously broadened lasers In Fig. 1(a), the inhomogeneity of medium $a$ is 2.5 . The pulse bandwidth is $\sim 1.3 \Delta \nu_{h, a}$, and the pulse shape shows noise spikes under a broad envelope duration of $\sim 8.66 t_{p 0}$. When the inhomogeneity of the gain medium is 5.0, the lasing spectrum has a larger bandwidth of $\sim 4.62 \Delta \nu_{h, a}$, as shown in Fig. 1(b). The pulse shape shows more noise spikes under a broader envelope with a duration of $\sim 11.39 t_{p 0}$.

With active mode locking of hybrid lasers, when the gain from the homogeneously broadened medium is added, the axial modes near the gain center will build up more favorably and tend to suppress the lasing of other axial modes through cross saturation of neighboring gains. As a result, the homogeneously broadened gain medium acts as an intracavity spectral filter to reshape the spectrum [10]. Fig. 2(a)-(d) show the spectra and pulse shapes of actively mode-locked hybrid lasers with different gain from medium $b$. Medium $a$ has an inhomogeneity of 2.5 and an unsaturated inversion ratio (ratio of the unsaturated
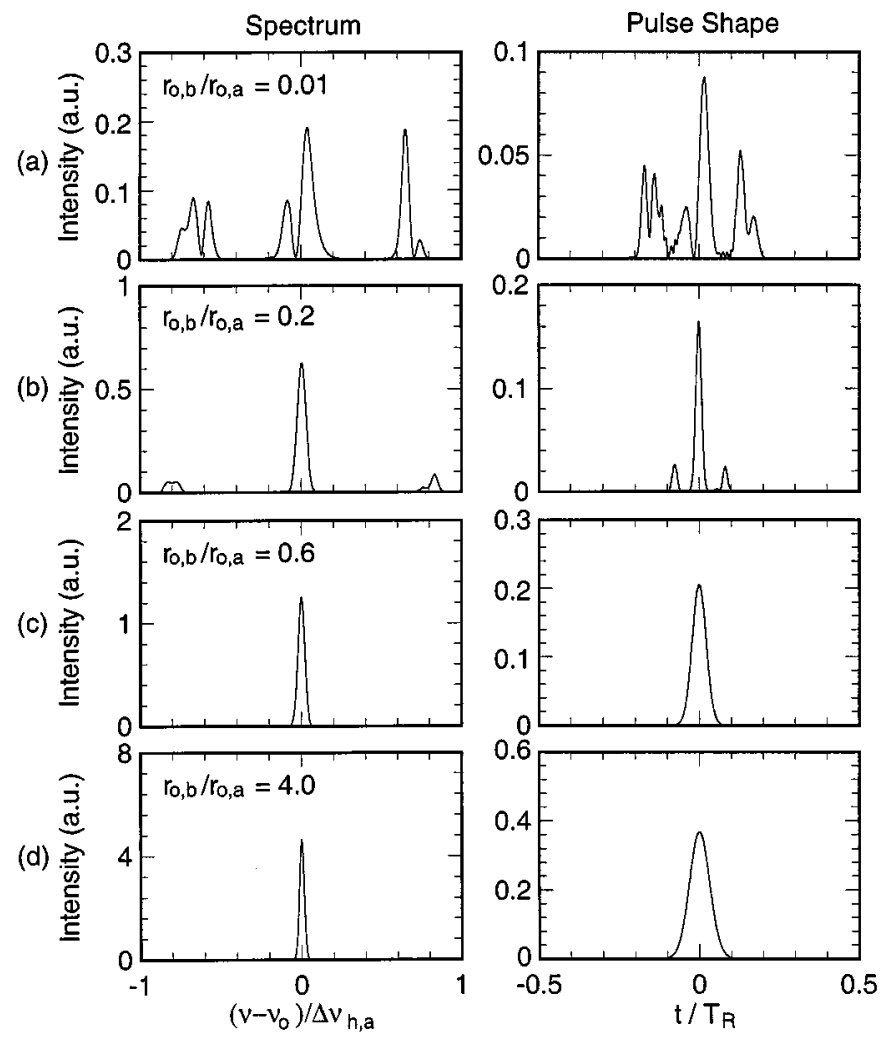

Fig. 2. Spectra and pulse shapes of an actively mode-locked hybrid laser with different gain from medium $b$. The unsaturated inversion ratio of medium $a$ is fixed at $r_{0, a}=5.0$. The inhomogeneity of medium $a$ is 2.5 . The modulation frequency and modulation index are $f_{m}=2 \mathrm{GHz}$ and $\Delta_{m}=0.00125$. The round-trip GVD is $k^{\prime \prime} p_{m}=2000 \mathrm{fs}^{2}$.

gain to the cavity loss) $r_{0, a}=5.0$. The unsaturated inversion ratios of medium $b$ is $r_{0, b}=0.05,1.0,3.0$, and 20.0, respectively. With $r_{0, b}=0.05$, as shown in Fig. 2(a), the spectral filtering by medium $b$ is weak. The spectrum is similar to that of the inhomogeneously broadened laser alone, and the pulse is partially coherent. With larger gain from medium $b$, as shown in Fig. 2(b) at $r_{0, b}=1.0$, the spectrum is reshaped with a clear peak at the center with pulse coherence improved, although unsuppressed spectral clusters exist in the outer spectral region. With sufficient gain from medium $b$, at $r_{0, b}=3.0$, a single peak spectrum with $\Delta \nu_{p}=0.0486 \Delta \nu_{h, a}$ appears, as shown in Fig. 2(c). Since this bandwidth is smaller than $\Delta \nu_{p 0}$, a coherent pulse is obtained. Note that this pulse bandwidth is about 1.6 times of that by using medium $b$ alone $\left(\sim 0.0296 \Delta \nu_{h, a}\right)$ under the same GVD and modulation magnitude. With even larger gain from medium $b$, the characteristics of the hybrid laser are more like that by using medium $b$ alone. For $r_{0, b}=20.0$, the pulse bandwidth is $\sim 0.0326 \Delta \nu_{h}, a$, about 1.1 times of that by medium $b$ alone, as shown in Fig. 2(d).

When medium $a$ has a stronger inhomogeneity, with $\Delta \nu_{i h, a} / \Delta \nu_{h, a}=5.0$, the spectral control feature in the hybrid laser is similar. As one expects, with a broader lasing bandwidth, more gain from the homogeneously broadened medium $b$ is required to suppress the lasing modes in the outer spectral region. Simulation shows that with the same GVD and modulation conditions, the gain from medium $b$ must be $r_{0, b}=20.0$ in order to have a single peak spectrum. The pulse 


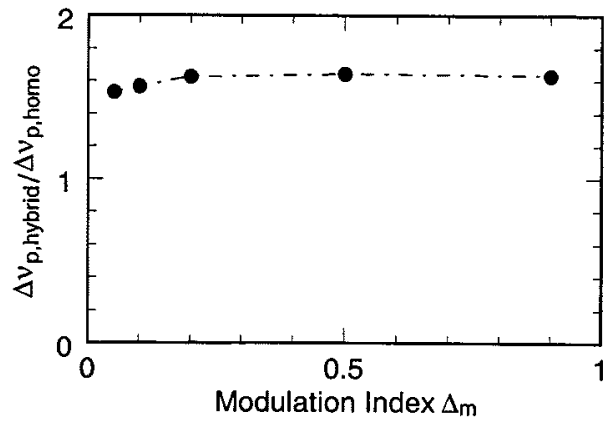

Fig. 3. Ratio of pulse bandwidths from a hybrid laser and a homogeneously broadened laser under different modulation index.

bandwidth is about 1.2 times that obtained by using medium $b$ alone.

The simulation results show two features of the actively mode-locked hybrid lasers. One is the spectral filtering by the narrow-band, homogeneously broadened medium. By using a second, narrow-band, homogeneously broadened medium, one can control the spectrum of a hybrid laser to within the lockable bandwidth by a modulator to obtain a coherent pulse. The second feature is that the pulse bandwidth of the actively mode-locked hybrid laser is larger than that of the narrow-band, homogeneously broadened medium alone. We observe these features because the gain narrowing effect is weaker in the hybrid laser than in the homogeneously broadened laser alone, as we will discuss in the next sub-section. These two features show the advantage of active mode locking with hybrid lasers over active mode locking with an inhomogeneously broadened medium, or a homogeneously broadened medium alone.

\section{B. Gain Characteristics}

As we noticed from the previous section, the pulse bandwidth obtained from the hybrid laser is $\sim 1.6$ times of that from the homogeneously broadened medium $b$ alone with $r_{0, a}=5.0$, $r_{0, b}=3.0$, and $\Delta_{m}=0.00125$ for $f_{m}=2 \mathrm{GHz}$ (equivalent to $\Delta_{m}=0.5$ for $f_{m}=100 \mathrm{MHz}$ ). We further studied this ratio, $\Delta \nu_{p}$, hybrid $/ \Delta \nu_{p}$, homo, under different modulation index $\Delta_{m}$, and the results are shown in Fig. 3. We see that $\Delta \nu_{p}$, hybrid $/ \Delta \nu_{p}$, homo is almost constant at $\sim 1.6$ when the modulation index $\Delta_{m}$ changes from 0.000125 to 0.00225 for $f_{m}=2 \mathrm{GHz}$ (equivalent to $\Delta_{m}$ changing from 0.05 to 0.9 for $f_{m}=100 \mathrm{MHz}$ ). Thus, the actively mode-locked hybrid laser behaves similarly to a homogeneously broadened laser because the overall gain profile is not affected under different modulation index. Since it is the curvature at the center of the saturated gain profile that determines the gain narrowing and, hence, the pulse bandwidth, one can conclude that the hybrid laser has a larger effective gain linewidth than the homogeneously broadened laser alone.

In a hybrid laser, the total gain is the sum of the gains from media $a$ and $b$. Fig. 4(a) and (b) show the unsaturated and saturated inversion ratio profiles of a hybrid laser with $r_{0, a}=$ 5.0 , and $r_{0, b} / r_{0, a}=0.6$. In the steady state, as shown in Fig. 4(b), the peak gain of medium $b$ is saturated down to a smaller and below threshold value. By the requirement of unity net gain, the gain of medium $a$ is also reduced to below threshold (a)

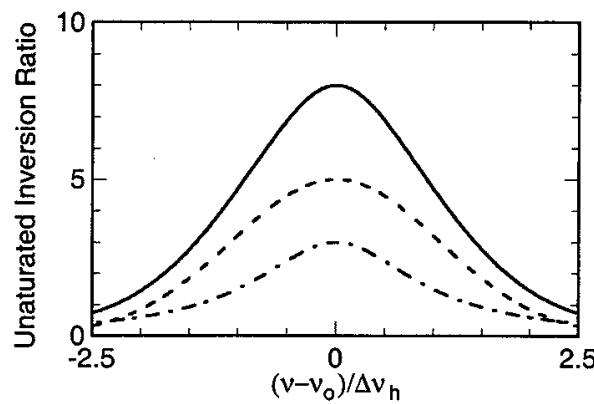

(b)

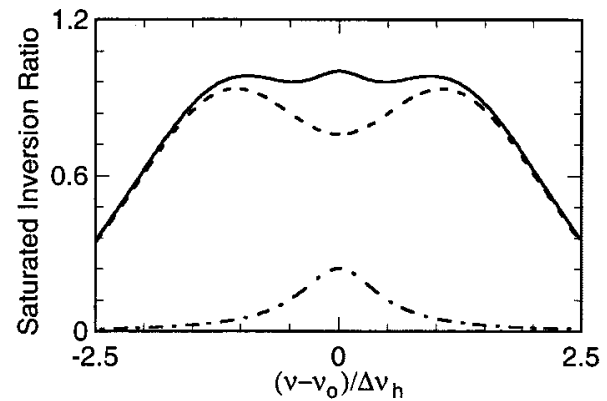

Fig. 4. (a) Unsaturated inversion ratios and (b) steady-state saturated inversion ratios of an actively mode-locked hybrid laser. The unsaturated inversion ratios for medium $a$ and $b$ are $r_{0, a}=5.0$ and $r_{0, b}=3.0$. The inhomogeneity of medium $a$ is 2.5. Dashed lines: medium $a$. Dashed-dotted lines: medium $b$. The solid lines are for the hybrid laser.

at the center. In the outer spectral region, the gain is mostly from medium $a$, which is less saturated by the center modes. Thus, the saturated gain profile of medium $a$ has a "hole" at the center, which has the same physical origin as the spectral hole burning. This "hole" compensates, to a large degree, the narrowly protruding gain profile of medium $b$, resulting in a flat overall gain profile which has a weaker curvature at the center than from gain medium $b$ alone. Therefore, the hybrid laser has weaker gain narrowing than the homogeneously broadened medium $b$, resulting in a broader pulse bandwidth under the same mode-locking condition.

It is easy to understand that the smaller the saturated gain of medium $b$ in the hybrid laser, the shallower the "hole," the flatter the overall gain profile, and the weaker the spectral filtering, resulting in a larger pulse bandwidth in active mode locking. However, partially coherent pulses are produced if lasing in the outer spectral region is not suppressed when the gain from medium $b$ is too small, as shown in Fig. 2.

\section{Influence of Parameters on the Pulse Coherence and Bandwidth}

As discussed previously, it is the saturated gain of medium $b$ that determines the flatness of the total gain profile and, thus, the degree of spectral filtering. With given media $a$ and $b$, one can adjust either the unsaturated gain or the saturation parameter of medium $b$ to influence its saturated gain.

Fig. 5 shows the pulse bandwidth of an actively mode-locked hybrid laser and the steady-state saturated gain of medium $b$ with different unsaturated gains contributed by medium $b$. The unsaturated gain of medium $a$ is fixed at $r_{0, a}=5.0$. The larger the unsaturated gain from medium $b$, the larger the steady-state saturated gain of medium $b$ and the stronger the gain filtering. Therefore, the pulse bandwidth becomes smaller and approaches the pulse 
(a)

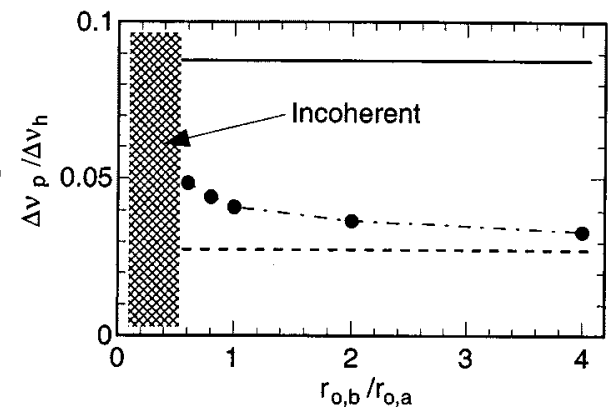

(b)

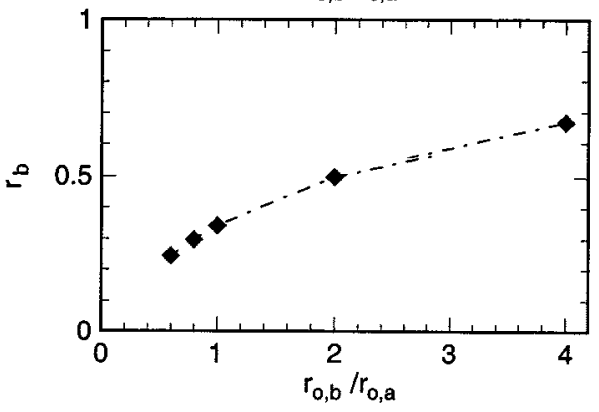

Fig. 5. (a) Normalized pulse bandwidth $\Delta \nu_{p} / \Delta \nu_{h, a}$ and (b) saturated inversion ratio $r_{b}$ of a hybrid laser under a different gain from medium $b$, with $r_{0, a}$ fixed at 5.0. The dashed line is the pulse bandwidth by medium $b$ alone, and the solid line is the maximum lockable pulse bandwidth with the same mode-locking strength and GVD.

bandwidth by medium $b$ alone. With smaller gain from medium $b$, the spectral filtering becomes weaker, and the pulse bandwidth becomes larger and reaches the maximal value at $r_{0, b} / r_{0, a}=$ 0.6 , just before the pulse becomes incoherent. The maximal pulse bandwidth is $\sim 1.6$ times of that by using medium $b$ alone, as can be seen from Fig. 5(a).

When the unsaturated gains from medium $a$ and medium $b$ vary but their ratio is kept the same, we find that the pulse bandwidth remains nearly the same. For example, for $r_{0, a}=1.25$, 2.5, 5.0, 10.0, and with $r_{0, b} / r_{0, a}=0.6$, the pulse bandwidths are almost the same as the corresponding point in Fig. 5(a). When we reach $r_{0, a}=20$, the pulse becomes incoherent. This can be explained as follows. The saturated inversion ratio of atom packet $\xi$ of medium $a$ is given by [10]

$$
r_{a}\left(\nu_{\xi}\right)=\frac{r_{0, a}\left(\nu_{\xi}\right)}{1+\sum_{n} \frac{I_{a}\left(\nu_{n}\right)}{I_{s, a}} \bar{g}_{a}\left(\nu_{\xi}, \nu_{n}\right)} .
$$

Since in the hybrid laser most of the power comes from the central modes, and $I_{a}\left(\nu_{n}\right)$ is proportional to the central gains $r_{0, a}\left(\nu_{\xi} \approx \nu_{0}\right)$ and $r_{0, b}$, the saturated gains $r_{a}\left(\nu_{\xi} \approx \nu_{0}\right)$ and $r_{b}$ remain nearly the same, resulting in the same saturated gain profile and the same pulse bandwidth. With very high pump levels, gains by atom packets farther away from the center frequency are not saturated down by the central modes, resulting in spectral clusters appearing in the outer spectral region. Consequently, coherent pulses can not be obtained, as for the case with $r_{0, a}=20.0$.

The saturated gain of medium $b$ is also influenced by the gain medium's saturation parameter. Since the intensity can be represented by $I_{a}\left(\nu_{n}\right) / I_{s, a}$, corresponding to the same intracavity (a)

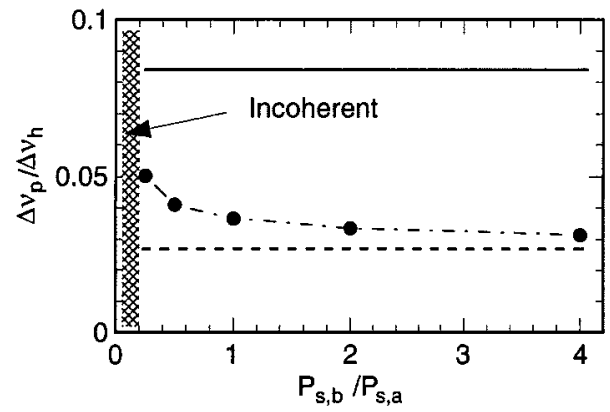

(b)

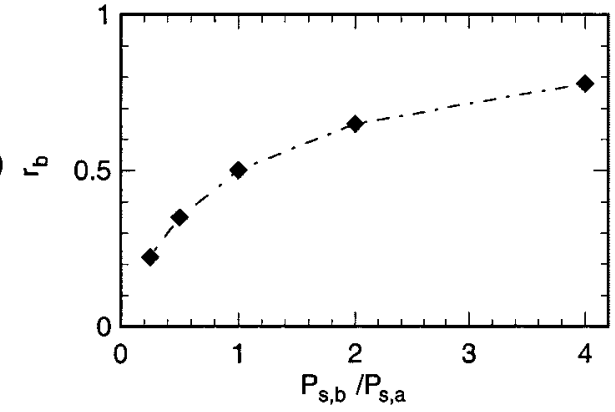

Fig. 6. (a) Normalized pulse bandwidth $\Delta \nu_{p} / \Delta \nu_{h, a}$ and (b) saturated inversion ratio $r_{b}$ of a hybrid laser with a different saturation power from medium $b$, with $r_{0, a}=5.0$ and $r_{0, b}=10.0$. The dashed line is the pulse bandwidth from medium $b$ alone, and the solid line is the maximum lockable pulse bandwidth with the same mode-locking strength and GVD.

power, we can rewrite $I_{b}\left(\nu_{n}\right) / I_{s, b}$ in (3) as

$$
\frac{I_{b}\left(\nu_{n}\right)}{I_{s, b}}=\frac{I_{a}\left(\nu_{n}\right)}{I_{s, a}} \frac{P_{s, a}}{P_{s, b}}
$$

where $P_{s, a}=I_{s, a} A_{a}$ and $P_{s, b}=I_{s, b} A_{b}$ are the saturation powers of gain media $a$ and $b$, and $A_{a}$ and $A_{b}$ are the beam areas in gain media $a$ and $b$, respectively. Thus the saturation power ratio $P_{s, b} / P_{s, a}$ is the influencing saturation parameter. A practical way to change this ratio is to vary the beam sizes when the two gain media are chosen.

Fig. 6 shows the pulse bandwidth of an actively mode-locked hybrid laser and the steady-state saturated gain of medium $b$ under different $P_{s, b} / P_{s, a}$. The unsaturated inversion ratios of media $a$ and $b$ are fixed at $r_{0, a}=5.0$ and $r_{0, b}=10.0$. With larger $P_{s, b} / P_{s, a}$, medium $b$ is more difficult to saturate, and the steady-state saturated gain is larger, as shown in Fig. 6(b). Therefore, the gain filtering is stronger, and the pulse bandwidth approaches the pulse bandwidth from medium $b$ alone. With smaller $P_{s, b} / P_{s, a}$, gain medium $b$ saturates more easily, and the spectral filtering becomes weaker. The pulse bandwidth becomes larger and reaches the maximal value at $P_{s, b} / P_{s, a}=$ 0.25 , just before the pulse becomes incoherent. The maximal pulse bandwidth is $\sim 1.6$ times of that by using medium $b$ alone.

In order to increase the degree of spectral filtering to obtain coherent pulses, one can either increase the unsaturated gain or the saturation power of medium $b$. The shaded area in Fig. 7(a) shows the region where coherent pulses can be obtained. The shortest pulses are obtained inside and near the boundary of the coherent region where the spectral filtering is the weakest while coherent pulses can still be obtained. The circles in Fig. 7(b) shows the corresponding maximal pulse bandwidths. One can see that the maximal pulse bandwidth is nearly constant, about 1.6 times the pulse 
(a)

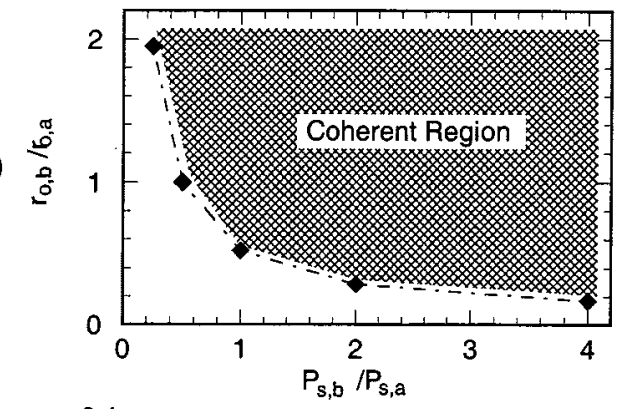

(b)

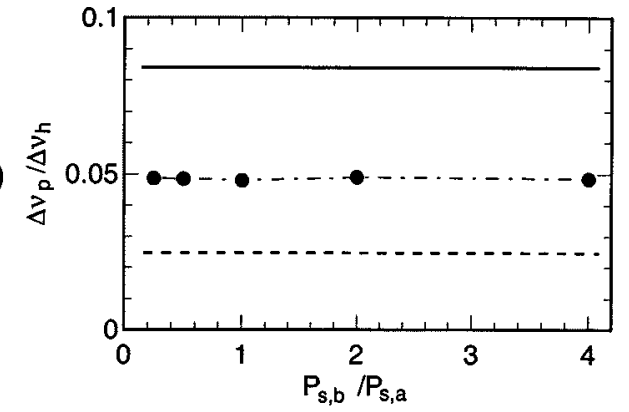

Fig. 7. (a) Coherent pulse region with a different gain ratio and saturation power ratio. (b) Normalized pulse bandwidth $\Delta \nu_{p} / \Delta \nu_{h, a}$ along the boundary in (a). Dashed line: pulse bandwidth from medium $b$ alone, and the solid line is the maximum lockable pulse bandwidth with the same mode-locking strength and GVD.

bandwidth obtained by using medium $b$ alone. This result is easy to understand. It is the steady-state saturated gain of medium $b$ that determines the degree of spectral filtering. When $r_{b}$ is saturated to the same optimal value, by reducing either the unsaturated gain or the saturation power, the shortest possible pulse that is still coherent is obtained.

When different gain media can be selected, the linewidth of medium $b$ is an important parameter that influences the spectral filtering in the hybrid lasers. Fig. 8(a) shows the pulse bandwidth obtained from actively mode-locked hybrid lasers using homogeneously broadened gain medium $b$ with different linewidth. The unsaturated gains from media $a$ and $b$ are fixed at $r_{0, a}=5.0$ and $r_{0, b}=20.0$, and both media $a$ and $b$ have the same saturation power. With small $\Delta \nu_{h, b}$, the spectral filtering is strong and the pulse bandwidth is about the same as that using medium $b$ alone. With increasing homogeneous linewidth, the spectral filtering becomes weaker, and the pulse bandwidth increases substantially. When $\Delta \nu_{h, b}$ is too large $\left(\Delta \nu_{h, b} / \Delta \nu_{h, a}>3.5\right.$ in this case), the spectral filtering is so weak that coherent pulses cannot be obtained. In Fig. 8(b), we show the corresponding steady-state saturated inversion ratio of medium $b$. One can see that $r_{b}$ is saturated down to nearly the same value for hybrid lasers with different $\Delta \nu_{h, b}$. We obtain this result because, with the same pump level, the laser power $\sum_{n} I\left(\nu_{n}\right)$ is about the same, no matter how large $\Delta \nu_{h, b}$ is. Since, for the hybrid laser, the lasing modes are near the center where $\bar{g}\left(\nu_{n}\right) \approx \bar{g}\left(\nu_{0}\right)=1$, the saturated inversion ratio of medium $b$, which is given by [10]

$$
r_{b}=\frac{r_{0, b}}{1+\sum_{n} \frac{I\left(\nu_{n}\right)}{I_{s}} \bar{g}\left(\nu_{n}\right)}
$$

is nearly the same for different $\Delta \nu_{h, b}$. (a)

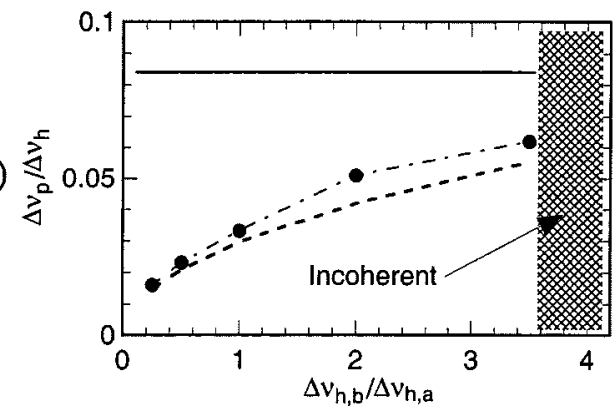

(b)

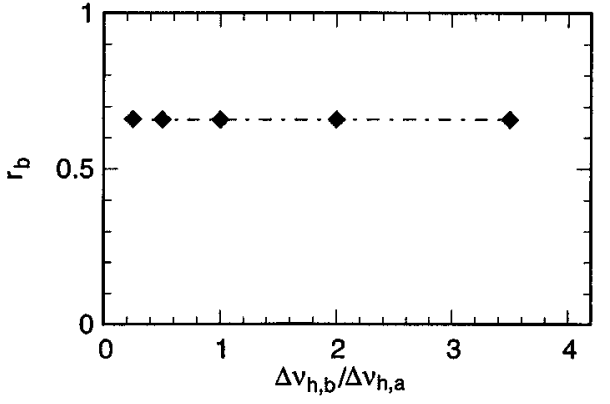

Fig. 8. (a) Normalized pulse bandwidth $\Delta \nu_{p} / \Delta \nu_{h, a}$ and (b) saturated inversion ratio $r_{b}$ of hybrid lasers with a different homogeneous linewidth in medium $b$ at $r_{0, a}=5.0$ and $r_{0, b}=20.0$. Dashed line: pulse bandwidth from medium $b$ alone, and the solid line is the maximum lockable pulse bandwidth under the same mode-locking strength and GVD.

One can adjust either the unsaturated gain ratio $r_{0, b} / r_{0, a}$ or the saturation power ratio $P_{s, b} / P_{s, a}$ to obtain the same maximal pulse bandwidth in active mode locking of a hybrid laser with a given $\Delta \nu_{h, b}$. In practical operation, however, adjusting $r_{0, b} / r_{0, a}$ is easier. The shaded area in Fig. 9(a) shows the region where coherent pulses can be obtained when one varies $\Delta \nu_{h, b}$ and $r_{0, b} / r_{0, a}$. We obtain the shortest pulses inside and near the boundary of the coherent region. Fig. 9(b) shows the corresponding maximal pulse bandwidth. For $\Delta \nu_{h, b} / \Delta \nu_{h, a}=$ 4.0 , the pulse bandwidth obtained from the hybrid laser can be $\sim 0.71$ times the GVD-limited pulse bandwidth $\Delta \nu_{p}$ under $r_{0, b} / r_{0, a}=9.0$. It is easy to understand that larger maximal pulse bandwidth can be obtained in a hybrid laser using medium $b$ with larger $\Delta \nu_{h, b}$. In the region with narrow gain linewidth $\Delta \nu_{h, b}$, most of the gain is from the inhomogeneously broadened medium and the homogeneously broadened medium acts as a spectral filter to help increase the pulse coherence. In the region with large gain linewidth $\Delta \nu_{h, b}$, most of the gain is from the homogeneously broadened medium, and the inhomogeneously broadened medium acts to further flatten the gain profile and yield a larger pulse bandwidth than when the medium is only homogeneously broadened.

Fig. 10 shows the ratio of maximal pulse bandwidth obtained from an actively mode-locked hybrid laser with $\Delta \nu_{h, b} / \Delta \nu_{h, a}=4.0$ and that from the homogeneously broadened laser with different GVDs. The trend is that, with a smaller dispersion, the increase of pulse bandwidth with the hybrid laser over the homogeneously broadened laser can be much larger. We obtain this result because, in a homogeneously broadened laser, the pulse bandwidth is mostly determined by the gain linewidth and this observation becomes more true as the GVD decreases. On the other hand, as the GVD decreases, the GVD-limited, maximum lockable bandwidth 
(a)

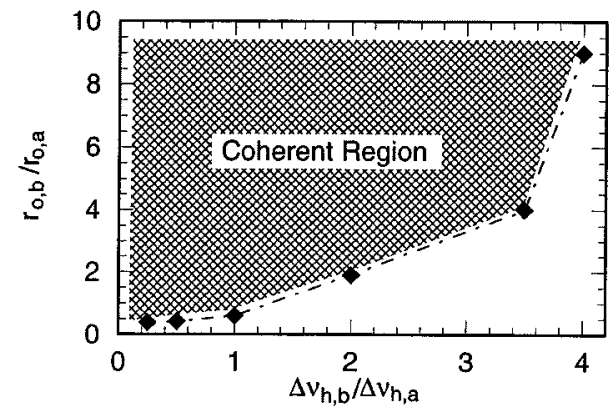

(b)

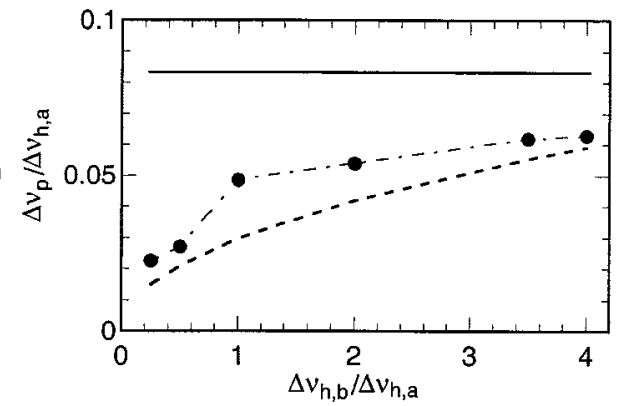

Fig. 9. (a) Coherent pulse region under different gain ratio and homogeneous linewidth of medium $b$. (b) Normalized pulse bandwidth $\Delta \nu_{p} / \Delta \nu_{h, a}$ along the boundary in (a). Dashed line: pulse bandwidth from medium $b$ alone. Solid line: maximum lockable pulse bandwidth with the same mode-locking strength and GVD.

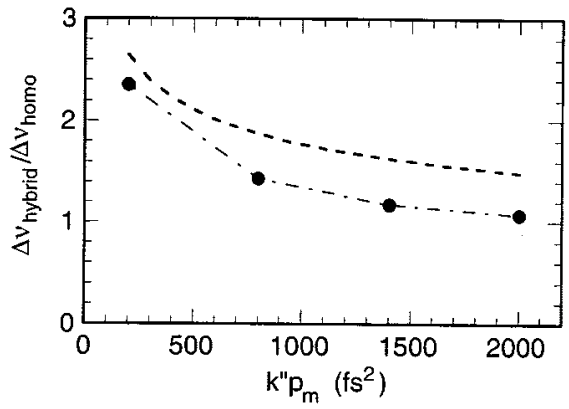

Fig. 10. Ratio of pulse bandwidths from a hybrid laser with $\Delta \nu_{h, b} / \Delta \nu_{h, a}=$ 4.0 and a homogeneously broadened laser with different GVDs. Dashed line: GVD-limited pulse bandwidth normalized to that from the homogeneously broadened laser.

$\Delta \nu_{p 0}$ increases, and only a little help from the hybrid scheme is needed to lock a broad bandwidth. Hence, the pulse bandwidth approaches more closely to $\Delta \nu_{p 0}$, and there is a larger improvement than with a homogeneously broadened laser alone.

\section{CONCLUSION}

In active mode locking of a laser, there exists a GVD-limited, maximum lockable bandwidth $\Delta \nu_{p 0}$ for a given modulation curvature $\Delta_{m} f_{m}^{2}$. For a homogeneously broadened laser, the pulse bandwidth is always smaller than $\Delta \nu_{p 0}$ due to the strong gain narrowing effect which limits the pulse duration. A broadband inhomogeneously broadened laser can usually sustain a lasing bandwidth larger than $\Delta \nu_{p 0}$ and consequently generates broad and partially coherent pulses.

In a hybrid laser, spectral filtering by the homogeneously broadened medium narrows the pulse bandwidth down to within the maximum lockable bandwidth, and coherent and shorter pulses are generated as compared to that by the inhomogeneously broadened gain medium alone. The overall gain profile in the hybrid laser is flatter than that of the homogeneously broadened medium, resulting in easier spectral broadening and generation of shorter pulses. With given gain media, varying the unsaturated gain or the saturation power of the gain medium are two equivalent ways to affect the gain saturation and to obtain the same maximal coherent pulse bandwidth. With the use of a broadband homogeneously-broadened medium, a larger pulse bandwidth can be obtained. The increase of the pulse bandwidth with the hybrid laser relative to the homogeneously broadened laser is much larger with smaller GVD.

The main features of the simulation results agree with the experimental observations from an actively mode-locked hybrid $\mathrm{Nd}$ : YLF and Nd : phosphate glass laser [3]: A small gain from the Nd: YLF medium can control and reshape the spectrum of the Nd: phophate glass and help generate coherent and shorter pulses, and the pulse bandwidth obtained from the hybrid $\mathrm{Nd}$ laser is about twice that of the Nd:YLF alone. The theoretical results provide an insight into the physics of the dynamical process of actively mode-locked hybrid lasers as well as a guidance of the construction and operation of hybrid lasers in active mode locking.

\section{REFERENCES}

[1] A. Girard, "The effects of the insertion of a cw, low-pressure $\mathrm{CO}_{2}$ laser into a TEA $\mathrm{CO}_{2}$ laser cavity," Opt. Commun., vol. 11, pp. 346-351, 1974.

[2] L. Yan and L. Ding, "Intracavity injection lasing in a hybrid neodymium laser," Appl. Phys. Lett., vol. 67, pp. 3679-3681, 1995.

[3] L. Yan and B. Guo, "Active mode locking with a hybrid neodymium laser," Appl. Phys. Lett., vol. 70, pp. 3501-3503, 1997.

[4] D. Kopf, F. X. Kartner, K. J. Weingarten, and U. Keller, "Pulse shortening in a Nd : glass laser by gain reshaping and soliton formation," Opt. Lett., vol. 19, pp. 2146-2148, 1994.

[5] L. Yan, J.-D. Ling, P.-T. Ho, C. H. Lee, and G. L. Burdge, "An actively mode-locked continuous wave Nd: Phosphate glass laser oscillator and regenerative amplifier," IEEE J. Quantum Electron., vol. 24, pp. 418-426, 1988.

[6] L. Yan, "Pulse coherence of actively mode-locked inhomogeneously broadened lasers," Opt. Commun., vol. 162, pp. 75-78, 1999.

[7] J. Zehetner, Ch. Spielmann, and F. Krausz, "Passive mode locking of homogeneously and inhomogeneously broadened lasers," Opt. Lett., vol. 17, pp. 871-873, 1992.

[8] F. Krausz, T. Brabec, and Ch. Spielmann, "Self-starting of passive mode locking," Opt. Lett., vol. 16, pp. 235-237, 1991.

[9] H. A. Haus and E. P. Ippen, "Self-starting of passively mode-locked lasers," Opt. Lett., vol. 16, pp. 1331-1333, 1991.

[10] L. Yan, "Continuous-wave lasing of hybrid lasers," IEEE J. Quantum Electron., vol. 33, pp. 1075-1083, 1997.

[11] A. E. Siegman, Lasers. Mill Valley, CA: Univ. Sci. Books, 1986, ch. 13 and 14.

Bo Guo was born in China in 1966. He received the B.S. and M.S. degrees from Beijing University, Beijing, China, both in physics, and the Ph.D. degree in electrical engineering from the University of Maryland at Baltimore County (UMBC) in 2001.

His research at UMBC included characterization of photorefractive crystals, numerical simulation, and experimental study of active mode locking with hybrid lasers. He was a Laser Scientist at Quantronix Corporation, East Setauket, NY, from 1997 to 2000, and a Senior Optical Engineer at Sycamore Network Inc. from 2000 to 2001. He is currently a Senior Optical Engineer with Celion Networks, Richardson, TX. 
Li Yan received the B.S. degree from the University of Science and Technology of China in 1982 and M.S. and Ph.D. degrees from the University of Maryland at College Park in 1986 and 1989, respectively.

After having worked as a Research Associate at the University of Maryland, in 1990 he joined the faculty of the Department of Electrical Engineering, the University of Maryland at Baltimore County, where he is currently an Associate Professor. His main research areas are ultrafast lasers, optical communications, and nonlinear optics.

Dr. Yan is a Member of the IEEE Lasers and Electro-Optics Society and the Optical Society of America.
Curtis R. Menyuk (SM'88-F'98) was born in 1954. He received the B.S. and M.S. degrees from Massachussetts Institute of Technology, Cambridge, MA, in 1976, and the Ph.D. from the University of California at Los Angeles in 1981.

He has been a Research Associate with the University of Maryland at College Park and Science Applications International Corporation, McLean, VA. In 1986, he was founding member and Associate Professor of the Department of Electrical Engineering, University of Maryland at Baltimore County (UMBC). In 1993, he was promoted to Professor. He is currently on partial leave and is Director of the Optical Networking Program at the Laboratory for Telecommunication Sciences, Adelphi, MD. For the last few years, his primary area of research has been theoretical and computational studies of fiber-optic communications. Computer codes that he wrote to model the nonlinear propagation of light in optical fibers have been used by industrial, government, and university research groups throughout the U.S. He has authored or co-authored more than 130 archival journal publications, as well as numerous other publications and presentations, and has edited two books.

Dr. Menyuk is a member of the Society for Industrial and Applied Mathematics and the American Physical Society. He is a Fellow of the Optical Society of America and is a UMBC Presidential Research Professor. 